\title{
TROMBOSIS DE VENAS Y SENOS VENOSOS CEREBRALES EN EL HOSPITAL DE SAN JOSÉ
}

Ramón Antonio Quintero Almenárez, MD*, Eduardo Palacios Sánchez, MD**, María Enoice Cifuentes Sánchez***, José Daniel Castro Avendaño.***

\section{Resumen}

Es un estudio descriptivo realizado para conocer las características clínicas y paraclínicas de los pacientes con trombosis de venas y senos venosos cerebrales que fueron hospitalizados, estudiados y manejados en el servicio de neurología del Hospital de San José. Se revisaron las historias clínicas de egresos de neurología y los archivos de estadística de los tres últimos años. Se hizo una descripción de los signos y síntomas presentados, se evaluó la presencia o ausencia de factores de riesgo, todos los casos se confirmaron con estudio imagenológico y se establecieron los factores que influyeron en el pronóstico. Estos resultados se comparan con lo descrito en la literatura mundial y nacional.

Palabras clave: trombosis venosa de senos cerebrales, resonancia magnética nuclear, fármacos antiepilécticos, angiorresonancia, angiografía, anticoagulación, estados protrombóticos, factores de riesgo.

Abreviaturas: TVSC, trombosis de venas y senos cerebrales; RMC, resonancia magnética cerebral; TAC, tomografía axial de cráneo; AR, angiorresonancia; LCR, líquido cefalorraquídeo.

\section{Introducción}

La TVSC es una condición poco frecuente pero con presentación clínica variable y a menudo dramática, que afecta en especial a personas jóvenes y mujeres en edad fértil. Aunque fue descrita por Ribes hace 180 años, solo desde hace poco menos de 30 años ha sido posible hacer el diagnóstico antemortem, en parte debido a la utilización de nuevas técnicas imagenológicas no invasivas.

La incidencia anual estimada es de tres a cuatro casos por millón en adultos, y siete por millón en niños. Cerca del $75 \%$ de pacientes adultos son mujeres. ${ }^{1}$

Fecha recibido: noviembre 17 de 2006-Fecha aceptado: diciembre 20 de 2006.

Residente IV de neurología. Fundación Universitaria de Ciencias de la salud, Hospital de San José, Bogotá, D.C.

** Profesor Titular de neurología. Fundación Universitaria de Ciencias de la Salud. Jefe Servicio de Neurología Hospital de San José, Bogotá D.C.

*** Estudiantes de medicina, Fundación Universitaria de Ciencias de la Salud. Hospital de San José, Bogotá, D.C.
La variabilidad de signos y síntomas se produce por dos mecanismos diferentes: 1) obstrucción venosa que ocasiona edema cerebral e infartos venosos y que explica los síntomas focales, y 2) trombosis de senos mayores que genera hipertensión endocraneana, producida por disminución del drenaje del LCR que explica los síntomas difusos.

En la mayoría de los casos hay compromiso de más de un seno venoso y la frecuencia aproximada de cada uno de estos es: seno transverso $86 \%$, sagital superior $62 \%$, recto $18 \%$, venas corticales $17 \%$, yugulares $12 \%$, vena de galeno y venas cerebrales internas $11 \%{ }^{2}$

Se han descrito cerca de cuarenta factores asociados con trombosis de senos venosos, entre los cuales se encuentran varias categorías: condiciones protrombóticas genéticas y adquiridas, infecciones, enfermedades inflamatorias, hematológicas, del colágeno, generales como la inflamatoria intestinal, medicamentos, traumas, procedimientos hemodinámicos y vasculares ${ }^{1,2,3}$ malaria, embarazo con niveles altos 
de homocisteinemia, punción lumbar y hemorragia subsaracnoidea. Sin embargo, en el 20 a $25 \%$ de los casos la causa no es identificada. 3,4

El estudio más sensible para el diagnóstico la TVSC es la RMC en combinación con la venografía con RM. Si la anterior no esta disponible, se puede utilizar TAC de preferencia de alta resolución o angio-TAC. Si los hallazgos de TAC o RMC, resultan inciertos, se requiere angiografía convencional.

Un alto porcentaje de pacientes con TVSC presenta secuelas como infartos venosos y hemorragia en $50 \%$, embolismo pulmonar $11 \%$, hipopituitarismo en la trombosis del seno cavernoso o crisis focales con y sin generalización secundaria. ${ }^{2}$

El objetivo de nuestro trabajo es conocer las características demográficas, comportamiento clínico, descripción de los factores de riesgo, tratamiento y secuelas encontradas en los pacientes con TVSC en el servicio de neurología durante los últimos tres años y compararlos con los análisis y descripciones de la literatura.

\section{Pacientes y métodos}

Es un estudio descriptivo retrospectivo documentado con imágenes de la población con TVSC hospitalizada en el servicio de neurología de Hospital de San José desde enero de 2003 hasta diciembre de 2005.

Se revisaron las historias clínicas de egreso con los siguientes criterios:

Criterios de inclusión: mayores de 18 años y TVSC documentada por imágenes (TAC. RM, angiografía).

Criterios de exclusión: pacientes con catéter venoso yugular y punción lumbar reciente.

\section{Resultados}

Se encontraron once pacientes de los cuales nueve $(81.8 \%)$ eran mujeres y dos (18.2\%) hombres, todos procedentes del área urbana con una edad promedio de 35 años; la mayoría eran jóvenes (nueve, 81.8\%). Tres (27.2\%) se presentaron de forma aguda (menos de 48 horas), seis ( $54.5 \%$ ) subaguda (48 horas a un mes) y dos (18.2\%) crónica (mayor a un mes).

Los síntomas por los cuales los pacientes consultaron al servicio de urgencias fueron cefalea en la mayoría (nueve $81.8 \%$ ), asociada con alteraciones del estado de conciencia (dos 18.2\%), alteraciones de las funciones mentales superiores (dos 18.2\%), náuseas o vómito (siete $63.6 \%$ ), fiebre (dos $18.2 \%$ ) y crisis convulsiva (dos $18.2 \%$ ) de los cuales uno inició con estatus convulsivo. Se presentaron alteraciones visuales en cuatro (36.4\%), motoras en tres $(27.2 \%)$, sensitivas en uno $(9 \%)$ y relajación de esfínteres en uno $(9 \%)$ (Gráfica 1).

Los signos encontrados en la valoración médica de ingreso fueron somnolencia (dos $18.2 \%$ ), desorientación uno $(9 \%)$ y déficit de pares craneales (cuatro, 36.4\%), cuya distribución fue: uno (II-VIII), dos (II), uno (III-VI), así como hemiparesia en dos $(18.2 \%)$.

En cuanto a los antecedentes personales descritos al ingreso fueron embarazo o puerperio cinco (45.4\%), cáncer uno $(9 \%)$, enfermedad del colágeno: dos $(18.2 \%)$, discrasias sanguíneas uno $(9 \%)$, coagulopatías tres $(27.2 \%)$, trombosis previas uno $(9 \%)$ y uso de anticonceptivos orales una (9\%).

El diagnóstico se confirmó con RM en cuatro pacientes $(36.4 \%)$, con AR en seis (54.5\%) y con angiografía en uno $(9 \%)$. La afección de los senos fue variable; se encontró compromiso de más de un seno en cinco casos (45.4\%), y única en seis (54.6\%). Las figuras 1 y 2 corresponden a trombosis de senos venosos en estudios de RMC y en la figura 3 observamos trombosis de seno venoso documentada por AR.

La distribución topográfica de la trombosis fue: una sagital superior y vena yugular, seis sagital superior, dos sagital superior y transverso, una transverso y vena yugular, una sagital superior, vena yugular, seno recto y seno cavernoso. (Gráfica 2 ). 
No. de pacientes

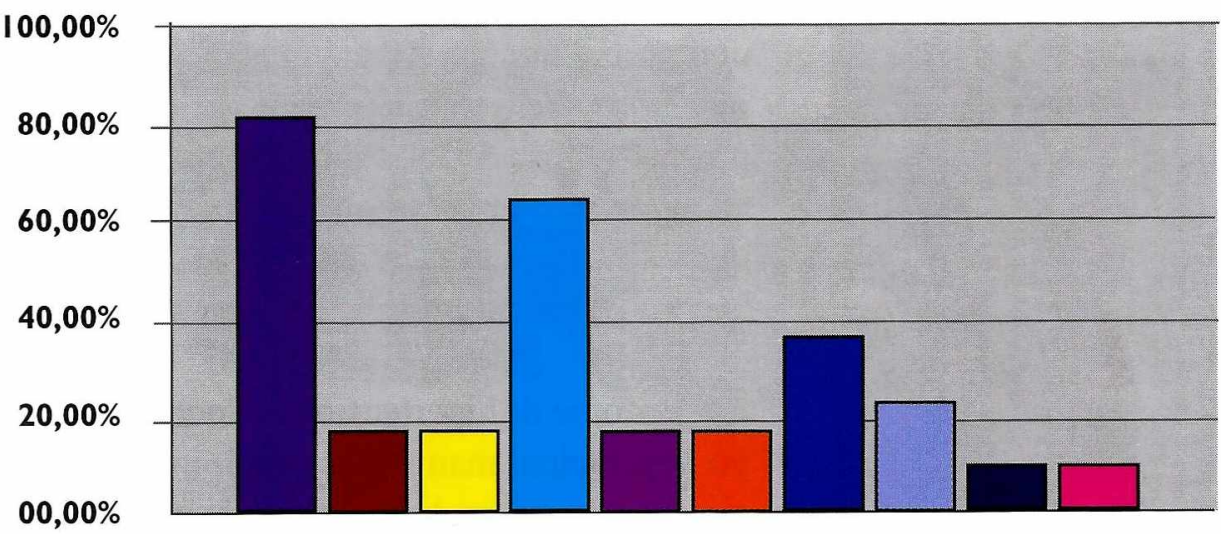

Cefalea

Alt. conciencia

Alt. mentales

$\square$ Náuseas/vómito

Fiebre

Crisis convulsivas

Alt. visuales

Alt. motoras

Alt. sensitivas

Alt. esfínteres

\section{Sintomas}

Gráfica I. Síntomas presentados en los pacientes con TSVC.

Otras anormalidades encontradas en los exámenes paraclínicos fueron dos en LCR (18.2\%), cinco con anticoagulante lúpico (45.4\%), una con anticardiolipínas $(9 \%)$, y cuatro con tiempos de coagulación alterados (36.4\%) (Tabla 1).

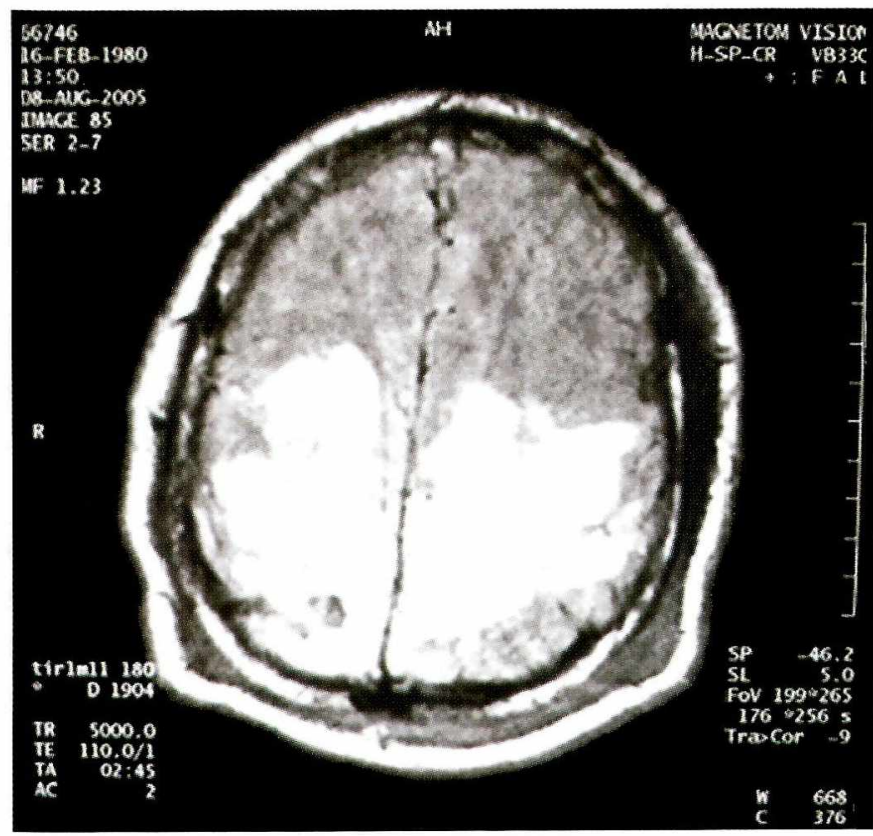

Figura I. Imagen en resonancia magnética nuclear de uno de nuestros pacientes donde se documentó TSVC.
$\mathrm{Al}$ egreso se encontró mejoría sin complicaciones en siete pacientes $(63.6 \%)$, otros presentaron complicación como infartos venosos (dos $18.2 \%$ ), crisis epilépticas recurrentes una (9\%), y cefalea uno (9\%) (Gráfica 3)

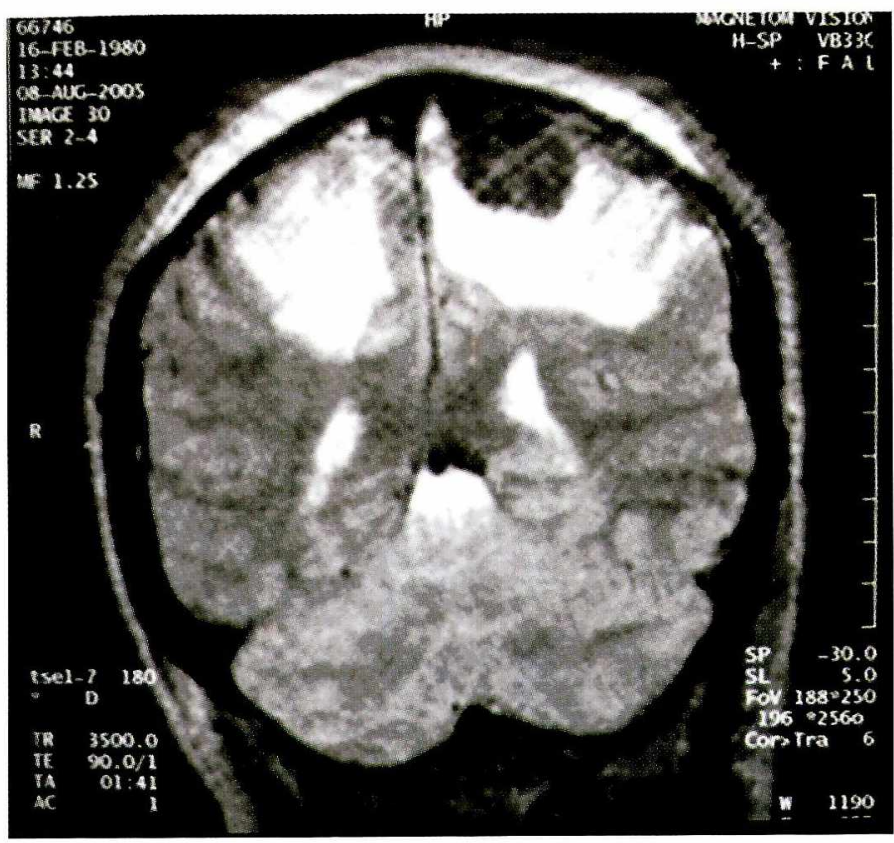

Figura 2. Corte coronal de resonancia magnética cerebral donde observamos el compromiso hemisférico bilateral producido por obstrucción del flujo venoso en TSVC. 


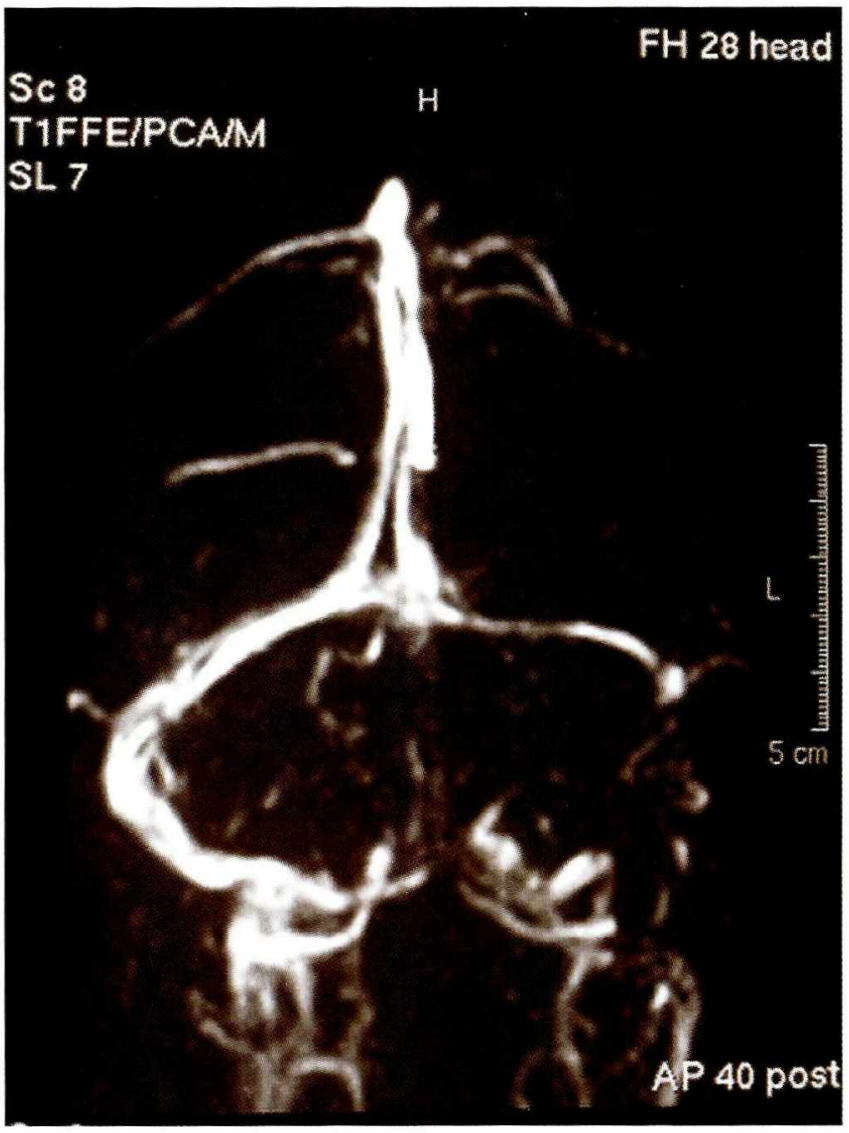

Figura 3. Angioresonancia magnética de una paciente de nuestra serie donde se evidencia TSVC.

\section{Discusión}

Todos los casos descritos de trombosis venosa fueron comprobados con estudio imagenológico. Debido a que los pacientes procedían del área urbana, no fue posible reconocer el comportamiento de la TSVC en las áreas rurales. Predominó el compromiso de pacientes jóvenes y de mujeres, lo cual está de acuerdo con lo descrito en la literatura. ${ }^{1}$

El síndrome de hipertensión endocraneana constituyó la principal manifestación al ingreso de los pacientes al servicio de urgencias, la mayoría de forma subaguda, lo cual también es similar a lo descrito en la literatura ${ }^{3}$ resultó interesante que solo dos tuvieron crisis, de los cuales uno inició con status de crisis focales que en forma secundaria se generalizaron; este mismo enfermo luego de 72 horas, presentó cuadriplejía flácida e hiperreflexia y se documentaron dos lesiones trombóticas diferentes una en seno sagital superior y la otra correspondiente a un infarto medular cervical. En cuanto a las anormalidades de los pares craneales, el mas afectado fue el segundo (2 pacientes) con papiledema.

Entre los factores de riesgo que predisponen a TVSC se destaca como importante el embarazo o

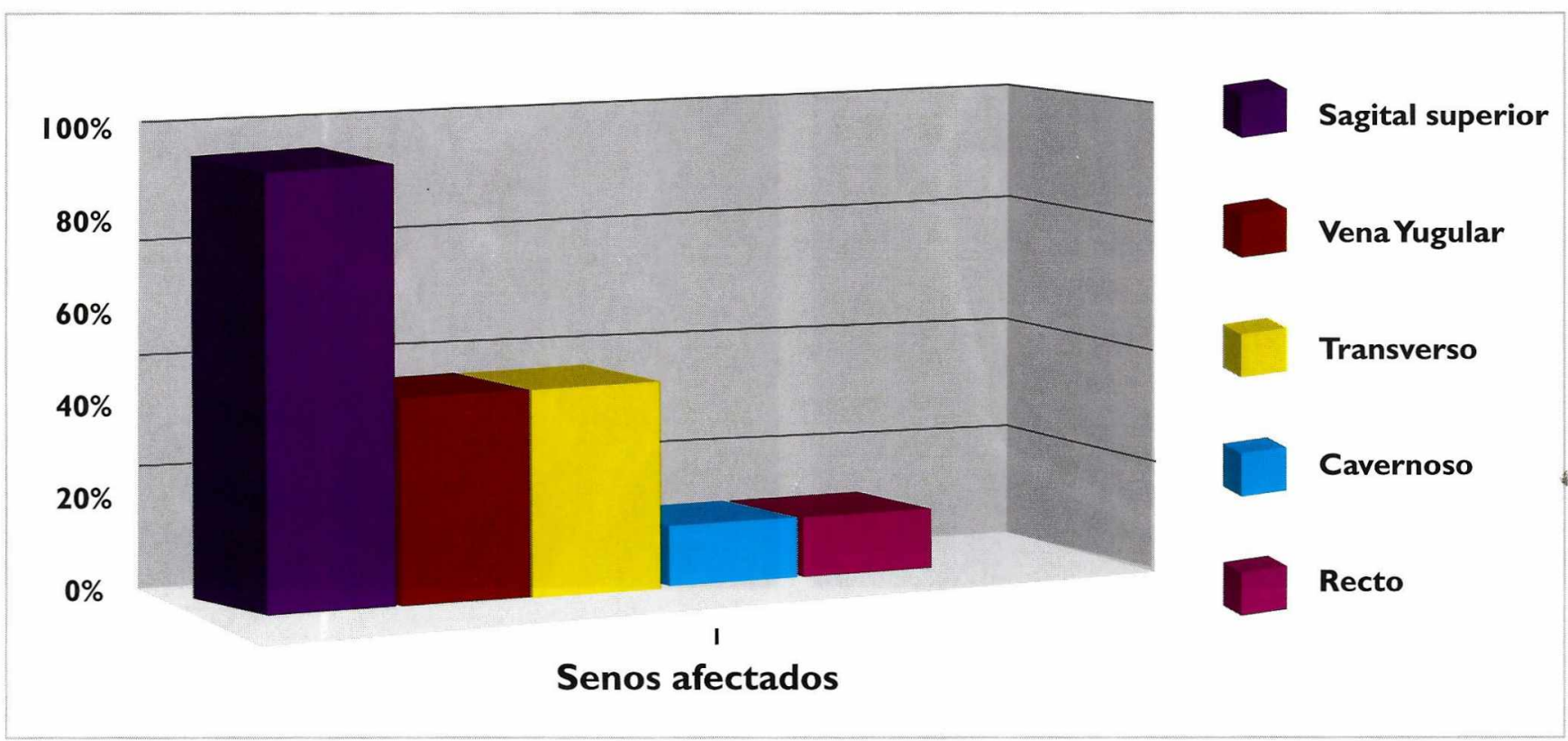

Gráfica 2. Se representan los principales senos y venas 


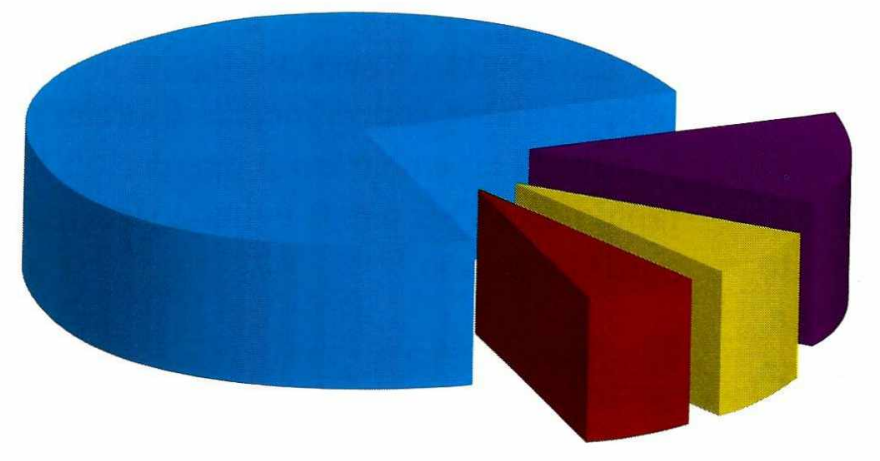

\section{Convulsiones}

Cefalea

- Infarto venoso

D Mejoría

Gráfica 3. Describe las complicaciones de los pacientes al egreso del manejo intrahospitalario.

puerperio1,5 lo cual también se observó en nuestro estudio, en $45.4 \%$ de los casos. Dos cursaban el primer trimestre gestacional, dos el tercero y una se encontraba en puerperio inmediato (dos días). En la literatura se describe con más frecuencia durante el tercer trimestre. ${ }^{1}$

Solo una paciente se relacionó con el uso de anticonceptivos orales (etinil estradiol $30 \mathrm{mcg}+$ levanorgestrel $150 \mathrm{mcg}$ ) factor ampliamente conocido. ${ }^{7,8}$ En otra paciente se documentó cáncer de ovario. Las coagulopatías estuvieron presentes en tres casos, cuyo tipo se encontraba en estudio. La literatura sugiere tener en cuenta los antecedentes familiares para el diagnostico. Así se documentaron dos con enfermedades del colágeno que correspondieron al síndrome antifosfolípidos y a artritis reumatoidea. E1 9\% de los pacientes tenían como antecedente haber padecido trombosis de la vena tibial posterior diez meses antes del episodio de TSVC. En ninguno se documentaron infecciones relacionadas con el evento.

La RMC fue el método diagnóstico en el $89 \%$ de los casos, lo cual refleja una sensibilidad alta, tal como está descrito1,9 solo un paciente requirió angiografía cerebral. Cerca de la mitad suelen tener afección de varios senos, lo cual también se corroboró en nuestro estudio, encontrando $45 \%$ de los casos con alteración de múltiples senos venosos y el 55\% con compromiso único; el sagital superior es el más afectado (89\%) contrario a lo encontrado por Stam, quien señala el seno recto.

El tratamiento de la TVSC está encaminado a eliminar el trombo, así como a eliminar la causa generadora. Se indica el manejo de la hipertensión endocraneana y el uso de anticonvulsivantes cuando sean necesarios. La eficacia de la heparina ha sido demostrada en varios estudios, llevando el tiempo parcial de tromboplastina hasta 1.5 a 2.0 veces el control. Se debe tener precaución cuando existe hemorragia intracerebral de gran tamaño, pero no es contraindicación para anticoagular a los pacientes con TSVC. En estos se lleva solo 1.25 a 1.5 veces el control. ${ }^{10,11,12}$ Se pueden

\begin{tabular}{|l|c|c|}
\hline \multicolumn{3}{|c|}{$\begin{array}{c}\text { Tabla } \\
\text { comprometidos con TSVC }\end{array}$} \\
\hline $\begin{array}{l}\text { ANORMALIDAD } \\
\text { PARACLÍNICA }\end{array}$ & No PACIENTES & $\%$ \\
\hline LCR & 2 & 18.2 \\
\hline Anticoagulante lúpico & 5 & 45.4 \\
\hline Anticardiolipinas & 1 & 9 \\
\hline Tiempo de coagulación & 4 & 36.4 \\
\hline
\end{tabular}


también utilizar heparinas de bajo peso molecular. ${ }^{13}$ La anticoagulación a largo plazo se recomienda por lo menos durante seis meses con warfarina. Cuando la etiología es infecciosa se requiere el uso de antibióticos, con especial énfasis en trombosis del seno cavernoso. El uso de esteroides es útil cuando la etiología está relacionada con lupus eritematoso sistémico o enfermedad de Behcet. ${ }^{14} \mathrm{El}$ tratamiento con anticonvulsivantes se recomienda con fenitoina, pero cuando hay crisis demostrables y no de manera profiláctica.

Cuando la hipertensión endocraneana es de difícil manejo, no se usa manitol ni furosemida. El drenaje de LCR se ha utilizado mediante punciones lumbares repetidas pero son riesgosas por el uso permanente de heparina. En algunas ocasiones es necesario realizar coma barbitúrico, fenestración del nervio óptico o derivación del liquido cefalorraquídeo a peritoneo cuando otras medidas no han sido efectivas. También se ha efectuado trombolisis con uroquinasa y activador del plasminógeno tisular recombínante. . $^{15,16,17}$ Sin embargo no existe suficiente evidencia para considerarlo como tratamiento de primera línea.

En general los resultados encontrados fueron similares a los descritos en otros estudios realizados en Colombia. ${ }^{18}$ Todos los pacientes documentados en nuestro estudio fueron anticoagulados con heparina y luego warfarina, tal como esta indicado en la literatura.1 Solo el paciente que inició con status persistió con crisis epilépticas y requirió fármacos anticonvulsivantes para su control. El $64 \%$ de los casos no tuvieron complicaciones y ninguno falleció.

\section{Conclusión}

En nuestra presentación comparamos la característica clínica, etiología y pronóstico, así como la utilidad de los medios diagnósticos disponibles, con lo publicado en la bibliografía mundial. Los resultados confirman que las características de nuestros pacientes no difieren sustancialmente de otros centros de atención terciaria; hay gran similitud de resultados con estudios realizados en nuestro país.
Se recomienda considerar la TVSC en pacientes con cefalea que no cumpla los criterios de la Sociedad Internacional de Cefalea para la forma primaria, en especial si está acompañada de signos de focalización o hipertensión endocraneana, sobretodo en jóvenes y mujeres en embarazo o puerperio.El diagnostico rápido de TVSC puede evitar consecuencias fatales para el enfermo.

\section{Referencias}

1. Stam J. Thrombosis of the cerebral veins and sinuses. N Engl J Med. 2005;352:1791-8.

2. Kimber J. Cerebral venous sinus thrombosis. QJM. 2002; 95:137-42.

3. Gozalez F, Saavedra M, Parra L, et al. Trombosis de senos venosos cerebrales. Acta Neurol Colomb. 2001;17:4251.

4. Allogren $\mathrm{H}$, Abbot R. Cerebral venous sinus thrombosis. Postgrad Med J 2000; 76(891):12-5.

5. Canhao P, Bousser MG, Barrigarrementeria $\mathrm{F}$ et al. Predisposing conditions for cerebral vein and dural sinus trombosis. Neurology. 2002; 249(supl 1): 178.

6. De bruijin S, Stam J, Koopman MM, et al. Case-control study of risk of cerebral sinus thrombosis in oral contraceptive users and in carriers of hereditary prothrombotic conditions. BMJ. 1998; 316:589-92.

7. Gillum LA, Mamidipudi SK, Johnston SC, et al. Ischemic stroke risk with oral contraceptives a meta-analysis. JAMA. 2000; 284:72-8.

8. Galidie G, Le Gall R, Cordoliani YS, et al. Thrombosis of the cerebral veins : X-ray computed tomographic and MRI imaging 11 cases. J Radiol.1992; 73(3):175-90.

9. Einhaupl K, Villringer A, Meister W, et al. Heparin treatment in sinus venous thrombosis. Lancet .1991; 338(8767):597-606.

10. Erbguth F, Brenner P, Schuierer G, Druschky KF et al. Diagnosis and treatment of deep cerebral vein thrombosis. Neurosurgery. 1991; 14:145-8.

11. Bousser MG, Barnett Hj. Cerebral venous thrombosis. En: Barnett HJ, Mor JP, Stein BM, Yatsu FM, editors. Stroke: pathophysiology, diagnosis and management. 3rd ed. New York: Churchill Livingstone; 1998. p. 623-47.

12. De Brujin SF, Stam J. For the cerebral venous sinus thrombosis study group. Randomized, placebo-controlled trial of anticoagulant treatment with low molecular-weigth heparin for cerebral sinus. Thrombosis. Stroke 1999; 30:484-8.

13. Weschler B, Dellsola B, Vidailhet M, et al. MRI in 31 patients with Behcets disease and neurological involvement: prospective study with clinical correlation .J Neurol Neurosurg Psychiatry. 1993; 56(7):793-8.

14. Barnwell S, Higasida R, Halbach V, et al. Direct endovascular thrombolytic theraphy for dural sinus thrombosis. Neurosurgery. 1991; 28:135-42. 
15. Eskridge JM, Wessbecher FW. Thrombolysis for superior sagittal sinus thrombosis. J Vasc Inter. Radiol. 1991;2:8994.

16. Horowitz M, Purdy P, Unwin H, et al. Treatment of dural sinus thrombosis using selective catheterization and urokinase. Ann Neurol. 1995; 38(1):58-67.
17. Volcy-Gomez M, Franco-Ruiz C, Uribe-Uribe C. Trombosis de senos venosos cerebrales: descripción de las características clínicas, factores de riesgo y tratamiento en un hospital de Colombia. Rev Neurol. 2003; 36:311-6.

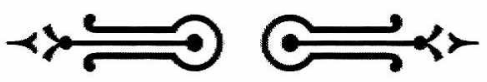

\section{FUNDACIÓN UNIVERSITARIA DE CIENCIAS DE LA SALUD HOSPITAL DE SAN JOSE}

Personería Jurídica No. 10917 Diciembre 1 de 1976 Resolución Ministerio de Educación Nacional No. 0125

Facultad de Instrumentación Quirúrgica

Código ICFES 270246100281100111100

Título otorgado Instrumentador Quirúrgico

Duración 8 semestres

No. créditos a cursar: 165

Modalidad: presencial

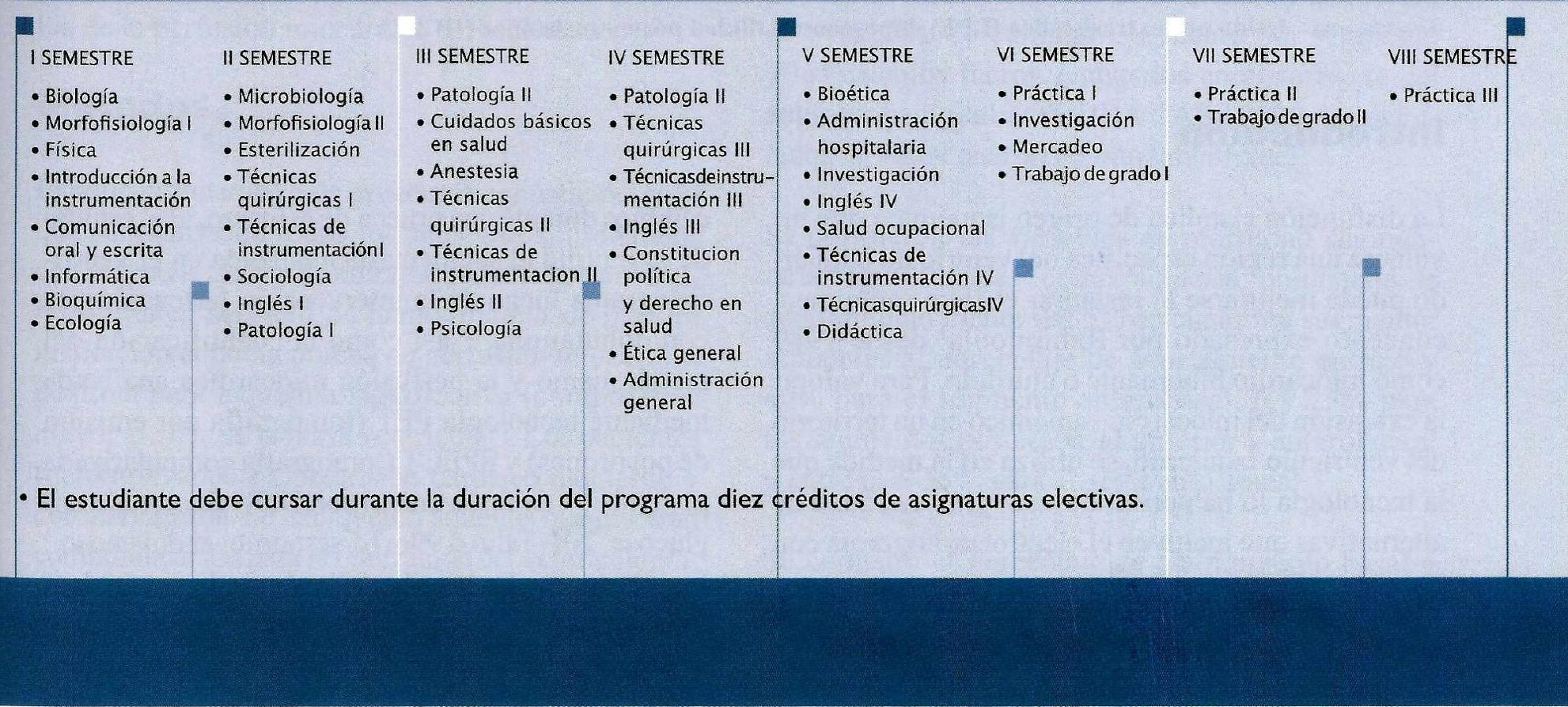

